FACTA UNIVERSITATIS

Series: Mechanical Engineering Vol. 14, N 3, 2016, pp. 281 - 292

DOI: $10.22190 / F U M E 1603281 \mathrm{~W}$

Original scientific paper

\title{
THE JKR-ADHESIVE NORMAL CONTACT PROBLEM OF AXISYMMETRIC RIGID PUNCHES WITH A FLAT ANNULAR SHAPE OR CONCAVE PROFILES
}

\author{
UDC 539.3
}

\author{
Emanuel Willert ${ }^{1}$, Qiang Li $^{1}$, Valentin L. Popov ${ }^{1,2,3}$ \\ ${ }^{1}$ Berlin University of Technology, 10623 Berlin, Germany \\ ${ }^{2}$ National Research Tomsk State University, 634050 Tomsk, Russia \\ ${ }^{3}$ National Research Tomsk Polytechnic University, 634050 Tomsk, Russia
}

\begin{abstract}
The JKR-adhesive frictionless normal contact problem is solved for the flat annular and the conical or spherical concave rigid punch indenting an elastic half space. The adhesive solution can be derived analytically from the non-adhesive one, the latter one being calculated by the boundary element method. It is found that the annular flat punch will always start to detach at the outer boundary. The pull-off forces for both concave punch shapes almost do not depend on the pull-off boundary regime and can be significantly larger than the pull-off force for the cylindrical flat punch.
\end{abstract}

Key Words: Contact Mechanics, Axis-symmetry, Annular Contact Area, Adhesion, JKR-theory, Boundary Element Method, Concave Rigid Punch, Flat Annular Punch

\section{INTRODUCTION}

Due to the ongoing miniaturisation of indenting devices in microscopy or material testing, adhesion in those systems is getting more and more important. Moreover, as the biological systems seem to have developed very efficient and powerful solutions for making use of adhesive interactions, the study of the contact mechanical interactions with their environment of insects, geckos - and other organisms relying on adhesion - has gained a lot of research interest in the past years. Spolenak et al. [1] found out that toroidal or concave shapes of the contact geometry - as they are used by these organisms - lead to a much better attachment of the indenting body to the surface. The contacting

Received September 27, 2016 / Accepted November 14, 2016

Corresponding author: Emanuel Willert

Institute of Mechanics, Berlin Institute of Technology, Strasse des 17. Juni 135, 10623 Berlin, Germany

E-mail: e.willert@tu-berlin.de 
bodies are usually considered to be soft, which is why the interaction range for the adhesion is small compared to the range of the elastic interaction. In this case the frictionless, adhesive normal contact problem can be solved by the theory developed by Johnson, Kendall and Roberts (JKR), [2]. The JKR-adhesive contact problem, though, can be turned back to the Boussinesq-problem, i.e. the frictionless non-adhesive normal contact problem of a rigid indenter pressed into an elastic half space.

Thereby many publications have dealt with the axisymmetric Boussinesq problem of an annular flat punch. Gubenko and Mossakovskij [3] and Collins [4] and [5] reduced the problem to an integral equation of the Fredholm type, which can be solved iteratively. For different approximate approaches see Borodachev and Borodacheva [6], Shibuya et al. [7] or Gladwell and Gupta [8]. A complete analytic - however recursive - solution was found by Roitman and Shishkanova [9]. A closed formulation could later be obtained by Antipov [10] using advanced applied mathematics including Riemann vector problems and Mellin transforms.

The Boussinesq problems for a conical or spherical concave rigid punch were tackled by Barber [11], Gladwell and Gupta [8] and Shibuya [12]. Barber - based on his idea presented earlier [13] that the actual contact area maximizes the normal force and can hence thereby be determined if not known a priori - gave series expansions of the solution for the ratio of the contact radii being close to zero or close to unity.

The JKR-adhesive problem for axisymmetric indenters and an annular contact area was first studied by Kesari and Lew [14]. Argatov et al. [15] demonstrated how the adhesive solution in this case can be obtained from the non-adhesive one and applied their method to conical concave (based on Barber's non-adhesive solutions) and toroidal (based on asymptotic non-adhesive solutions for narrow contact areas) indenters.

In the present paper we will analyze the JKR-adhesive normal contact problem of an axisymmetric annular flat or either conical or spherical concave rigid punch. The nonadhesive solutions presented in Section 2 are obtained via fast boundary element method (BEM) simulations - the fundamentals of which were described by Pohrt and Li [16] and will be approximated by simple analytic expressions. Afterwards in Section 3 the adhesive solutions are derived analytically from these non-adhesive results. Section 4 will give conclusions.

\section{THE NON-ADHESIVE SOLUTION}

We consider the Boussinesq problem for an annular flat or concave rigid punch pressed into an elastic half space. The normal force shall be $F_{N}$, the indentation depth $d$ and the inner and outer contact radii $b$ and $a$, respectively. The hole has depth $h$. The concave profile shall be either conical or spherical. Sketches of the problems considered and notations are shown in Fig. 1, 2 and 3.

In the case of the annular flat punch it is shown that the normal force can be written in the form:

$$
F_{N}=2 E^{*} d a \gamma(\varepsilon)
$$

with effective Young's modulus $E^{*}$ and the ratio of the contact radii:

$$
\varepsilon=\frac{b}{a} \text {. }
$$


As the closed form analytical solution given by Antipov [10] is hard to handle, we calculated it again using boundary element simulations. Function $\gamma(\varepsilon)$ can be approximated by the expression:

$$
\gamma(\varepsilon) \approx\left(1-\varepsilon^{m}\right)^{n}
$$

with:

$$
m \approx 2.915, \quad n \approx 0.147,
$$

obtained via a simple least-squared-error, gradient-based parameter optimization. The results of the BEM calculations together with the perfectly fitting analytical approximation are shown in Fig. 4.

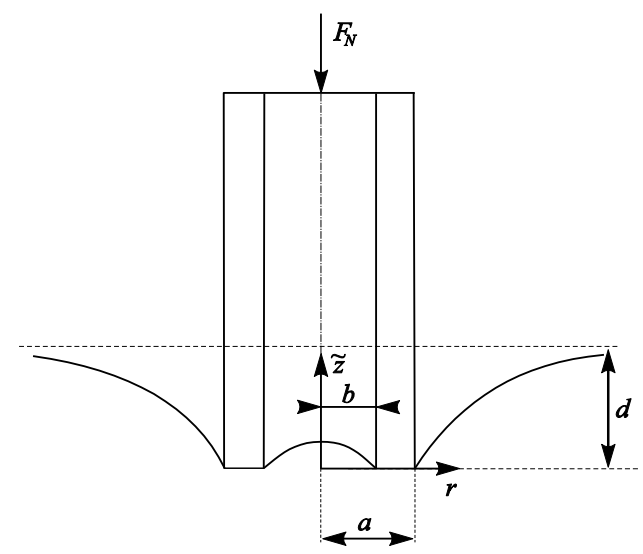

Fig. 1 Cross section of an annular flat rigid punch indenting an elastic half space

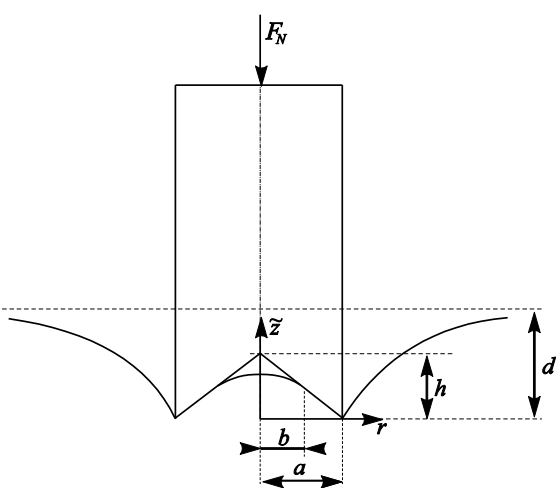

Fig. 2 Cross section of a conical concave rigid punch indenting an elastic half space

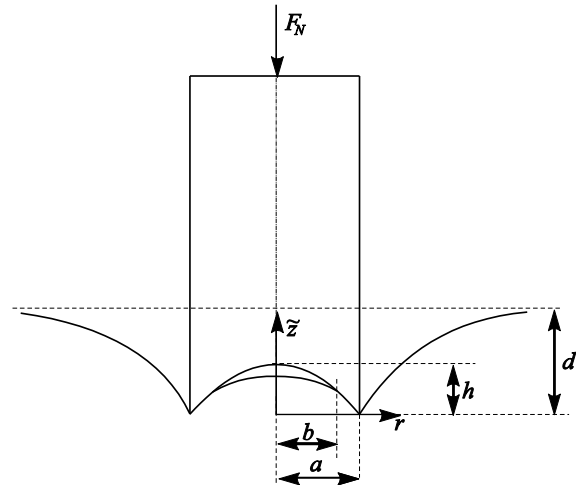

Fig. 3 Cross section of a spherically concave rigid punch indenting an elastic half space 


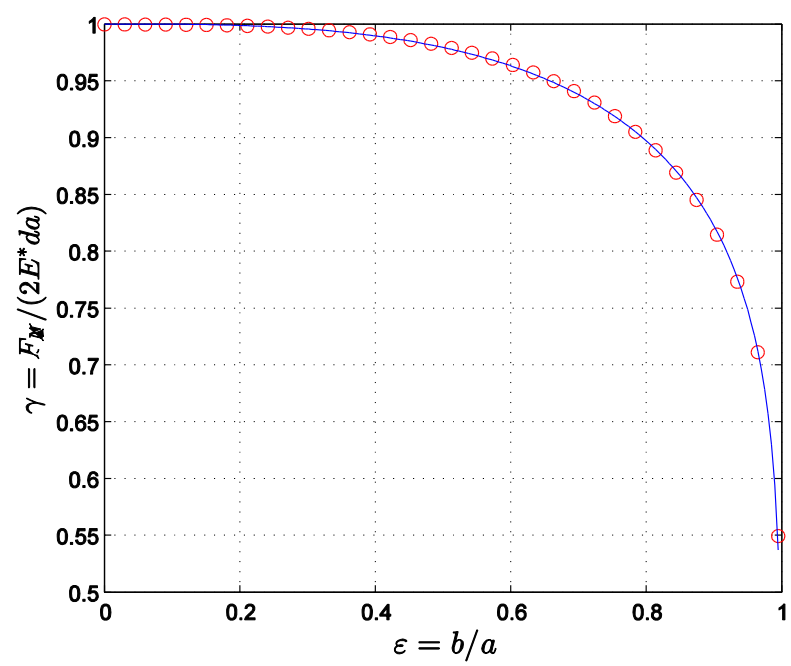

Fig. 4 Results of BEM simulations (circles) and analytic approximation (3) (solid line) for normalized normal force $\gamma=F_{N} / 2 E^{*} d a$, as a function of the ratio of contact radii $\varepsilon=b / a$ for the frictionless, non-adhesive indentation of an elastic half space by an annular flat rigid punch

In the case of a conical or spherical concave rigid indenter the inner contact radius $b$ is not fixed but depending on the indentation depth or the normal force. If we put $\varepsilon$ as the governing parameter for the contact problem, the solution in either case can be written in the form:

$$
\begin{aligned}
d & =h \gamma_{1}(\varepsilon), \\
F_{N} & =2 E^{*} h a \gamma_{2}(\varepsilon) .
\end{aligned}
$$

Barber [11] gave solutions for dimensionless functions $\gamma_{1}$ and $\gamma_{2}$ in the cases of $\varepsilon$ being close to zero or close to unity. For the conical hole results of our BEM calculations are shown in Fig. 5. Apparently it is:

$$
\gamma_{1}^{c o n}(\varepsilon) \approx \gamma_{2}^{c o n}(\varepsilon)
$$

and hence:

$$
F_{N} \approx 2 E^{*} d a,
$$

i.e. the indenter almost behaves like a rigid flat cylindrical punch. This is especially true for values of $\varepsilon$ close to zero. Note that $\varepsilon=0$ can never be reached as this would require an infinite normal force [11]. 


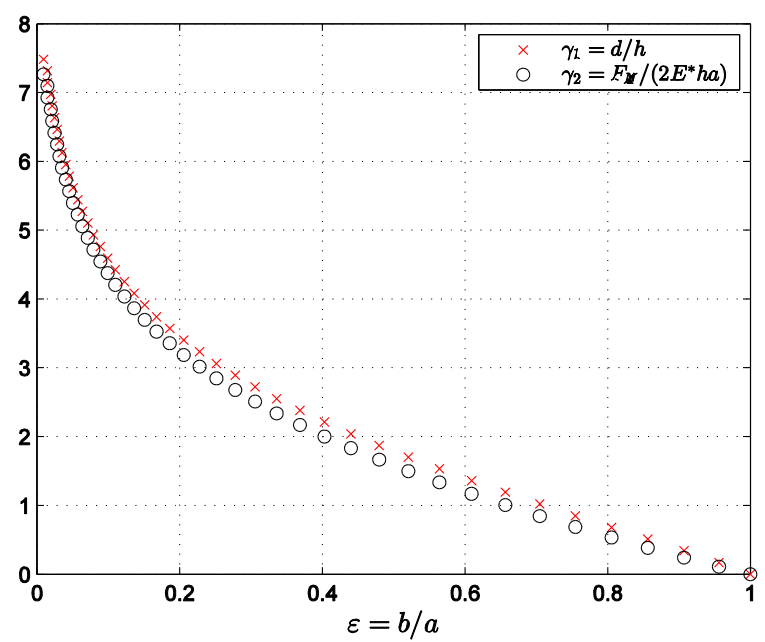

Fig. 5 Results of BEM simulations for normalized indentation depth $\gamma_{1}=d / h$ (crosses) and normal force $\gamma_{2}=F_{N} / 2 E^{*} h a$ (circles), as functions of the ratio of contact radii $\varepsilon=b / a$ for the frictionless, non-adhesive indentation of an elastic half space by a conical concave rigid punch

In more detail, both conical solutions can be approximated in the form:

$$
\gamma_{i}^{c o n}(\varepsilon) \approx(-\ln \varepsilon)^{n_{i}}\left(a_{i}+b_{i} \varepsilon+c_{i} \varepsilon^{2}\right), \quad i=1,2,
$$

with the fitted parameters:

$$
\begin{aligned}
& n_{1}=0.874, \quad a_{1}=2.09, \quad b_{1}=0.54, \quad c_{1}=0.15 \\
& n_{2}=0.945, a_{2}=1.85, \quad b_{2}=0.45, \quad c_{2}=0.12
\end{aligned}
$$

For the spherical concave indenter the solutions can be approximated very well by the functions:

with the parameters:

$$
\gamma_{i}^{s p h}(\varepsilon) \approx a_{i}\left(1-\varepsilon^{m_{i}}\right)^{n_{i}}, \quad i=1,2
$$

$$
\begin{aligned}
& a_{1}=3, m_{1}=2.034, n_{1}=0.91 \\
& a_{2}=8 / 3, m_{2}=2.015, n_{2}=1.03 .
\end{aligned}
$$

Note, that it can be easily proven analytically - for the spherical concave indenter - that full contact, i.e. $b=0$, is established for $d=3 h$ and $F_{N}=16 / 3 \cdot E^{*} h a$ [11]. Parameters $a_{i}$ in Eqs. (11) therefore have not been object to optimization.

The results of the BEM calculations for both concave indenter profiles together with the fitted analytic approximations from Eqs. (8) and (10) are shown in Fig. 6. 


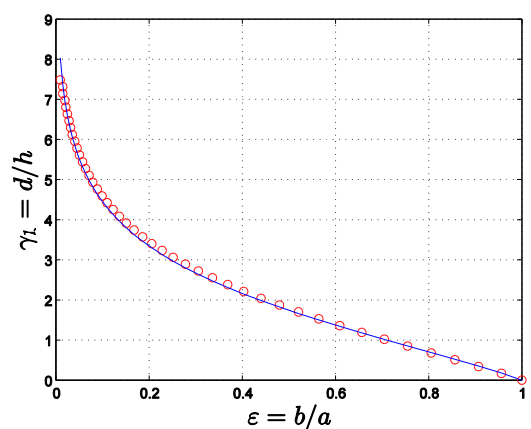

(a)

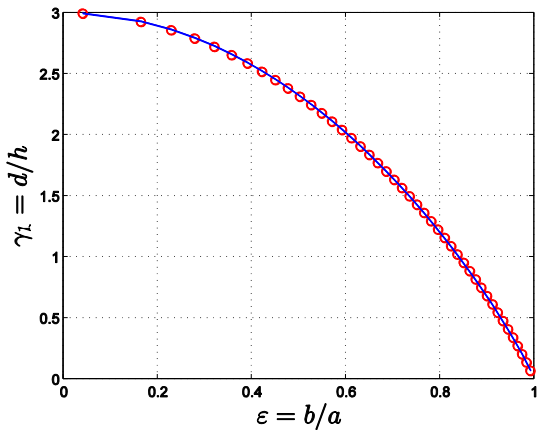

(c)

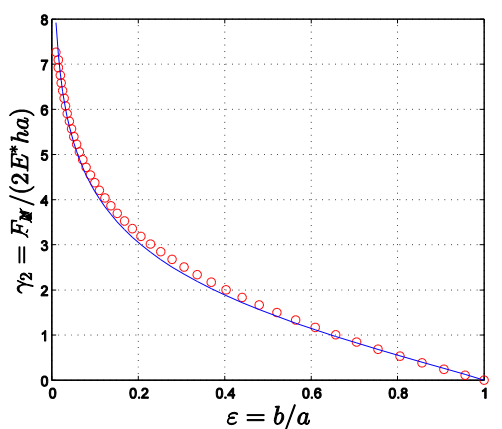

(b)

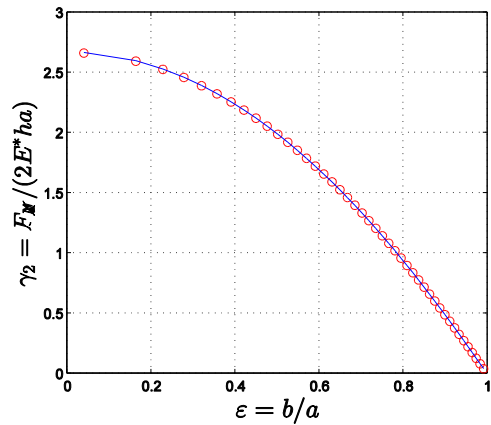

(d)

Fig. 6 BEM simulations (circles) and analytic approximations (solid lines) for normalized indentation depth $\gamma_{1}=d / h$ and normal force $\gamma_{2}=F_{N} / 2 E^{*} h a$ as functions of the ratio of contact radii $\varepsilon=b / a$ for the frictionless, non-adhesive indentation of an elastic half space by an either conical or spherical concave rigid punch:

(a) $\gamma_{1}$, conical (b) $\gamma_{2}$, conical (c) $\gamma_{1}$, spherical (d) $\gamma_{2}$, spherical

\section{THE ADHESIVE SOLUTION}

In the following we will denote the non-adhesive solutions by an upper index "na". For example, $F_{N}^{\text {na }}$ shall be the normal force given above for the non-adhesive problem, whereas $F_{N}$ shall be the full adhesive normal force.

Let us again consider the annular flat punch first. The non-adhesive normal force as a function of the non-adhesive indentation depth was given in Eq. (1). Within the JKRtheory the adhesion is modeled via an additional energy term:

$$
U_{a d}=-A \Delta w,
$$

with contact area $A$ and effective surface energy per unit area $\Delta w$. Hence, the total energy will be:

$$
U_{t o t}=U_{e l}+U_{a d}=E^{*} d^{2} a \gamma(\varepsilon)-\pi a^{2}\left(1-\varepsilon^{2}\right) \Delta w .
$$


The normal force is given by the derivative:

$$
F_{N}=\frac{\partial U_{t o t}}{\partial d}=2 E^{*} d a \gamma(\varepsilon) .
$$

As the contact radii are fixed and not connected to the indentation depth, this is the same relation as in the non-adhesive case. The contact loses its stability and detaches at the outer boundary $r=a$, if:

$$
\left.\frac{\partial U_{t o t}}{\partial a}\right|_{d=d_{c}}=E^{*} d_{c}^{2}\left[\gamma(\varepsilon)-\varepsilon \gamma^{\prime}(\varepsilon)\right]-2 \pi a \Delta w=0,
$$

from which we deduce the critical indentation depth:

$$
d_{c}=-\frac{d_{0}}{\sqrt{\gamma(\varepsilon)-\varepsilon \gamma^{\prime}(\varepsilon)}},
$$

and the critical adhesion force:

$$
F_{c}=-\frac{F_{0} \gamma(\varepsilon)}{\sqrt{\gamma(\varepsilon)-\varepsilon \gamma^{\prime}(\varepsilon)}}
$$

with the respective values for the flat cylindrical punch of radius $a$ obtained by Kendall [17]:

$$
d_{0}=\sqrt{\frac{2 \pi a \Delta w}{E^{*}}}, F_{0}=\sqrt{8 \pi a^{3} E^{*} \Delta w} .
$$

The condition for detachment starting at the inner boundary $r=b$ is analogously given by the relation:

$$
\left.\frac{\partial U_{t o t}}{\partial b}\right|_{d=d_{c}}=E^{*} d_{c}^{2} \gamma^{\prime}(\varepsilon)+2 \pi b \Delta w=0
$$

rom which we obtain the critical indentation depth:

$$
d_{c}=-d_{0} \sqrt{\frac{\varepsilon}{-\gamma^{\prime}(\varepsilon)}} .
$$

Derivative $\gamma^{\prime}(\varepsilon)$ is always negative so this indeed will be a real length. As it can be seen in Fig. 7 the absolute value of the critical indentation depth is always smaller for the detachment at the outer boundary. Thus, the contact detachment will indeed start there. 


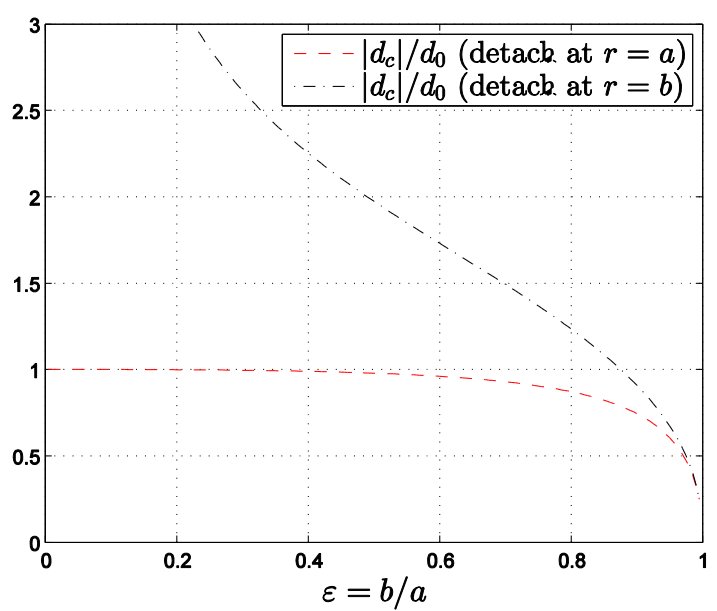

Fig. 7 Critical normalized indentation depths for the detachment at the inner $(r=b)$ and outer $(r=a)$ boundary for the JKR-adhesive normal contact of a rigid annular flat stamp

Let us compare our results to analytical calculations obtained earlier. For small values of $\varepsilon$ Collins [4] gives the analytical series expansion:

$$
\gamma(\varepsilon)=1-\frac{4}{3 \pi^{2}} \varepsilon^{3}-\frac{8}{15 \pi^{2}} \varepsilon^{5}-\frac{16}{27 \pi^{4}} \varepsilon^{6}+O\left[\varepsilon^{7}\right],
$$

which can be used in Eqs. (16) and (17) to calculate the critical contact configuration. For narrow contact areas, i.e. $\varepsilon$ near unity, Argatov et al. [15] gave the asymptotic expressions:

$$
\begin{aligned}
& d_{c} \approx-2 \ln \frac{16}{\delta} \sqrt{\frac{(a+b) \Delta w}{\pi E^{*}} \delta}, \\
& F_{c} \approx-\sqrt{\pi^{3}(a+b)^{3} E^{*} \Delta w \delta},
\end{aligned}
$$

with the transformed (small) variable:

$$
\delta=\frac{1-\varepsilon}{1+\varepsilon} .
$$

In Fig. 8 the results of Argatov et al. [15] for $\varepsilon>0.85$ together with the series expansion (21) used in the relations (16) and (17) are shown as dashed lines. Also the results, if the approximation (3) is used for Eqs. (16) and (17), are given as solid lines. Obviously, the curves overlap nicely for the limiting cases. The approximation obtained by non-adhesive BEM calculations also shows the transition behavior for intermediate values of $\varepsilon$, for which the two analytical but asymptotic approaches do not agree with each other very well. Thereby it has to be pointed out that the sixth-order series expansion by Collins gives very good results for approximately $\varepsilon<0.6$. 


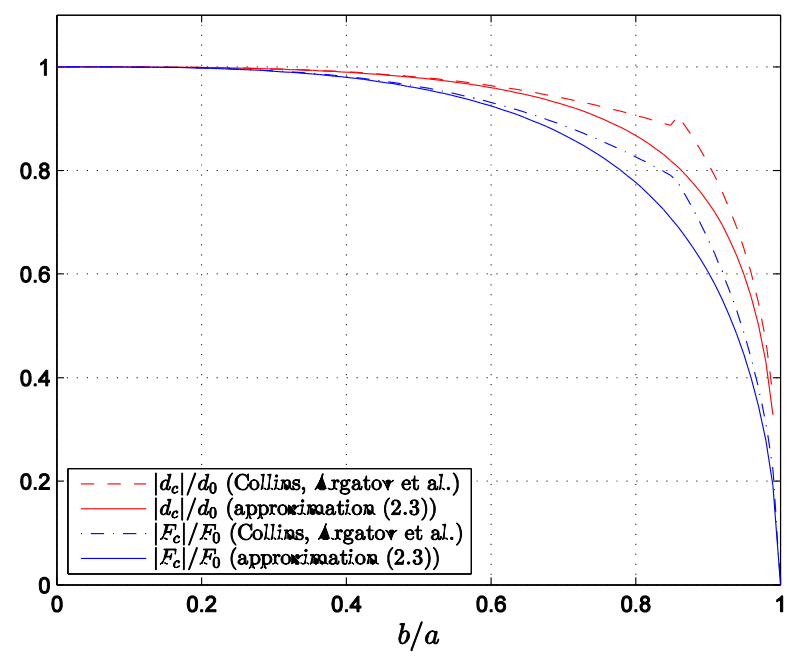

Fig. 8 Normalized critical indentation depth (red) and normal force (blue) as a function of the ratio of contact radii $\varepsilon=b / a$. Dashed: analytical results by Collins [4] (together with Eqs. (16) and (17)) for $\varepsilon<0.85$ and by Argatov et al. [15] for $\varepsilon>0.85$. Solid line: approximation (3) together with Eqs. (16) and (17)

To solve the adhesive problem for the concave indenters we recall the non-adhesive solution from Eq. (5):

$$
d^{\mathrm{na}}=h \gamma_{1}(\varepsilon), F_{N}^{\mathrm{na}}=2 E^{*} h a \gamma_{2}(\varepsilon) .
$$

Thereby one can define the non-adhesive contact stiffness:

$$
k_{N}^{\mathrm{na}}(\varepsilon)=\frac{\mathrm{d} F_{N}^{\mathrm{na}}}{\mathrm{d} d^{\mathrm{na}}}=2 E^{*} a \frac{\gamma_{2}^{\prime}(\varepsilon)}{\gamma_{1}^{\prime}(\varepsilon)},
$$

the prime denoting a derivative. According to Argatov et al. [15] the adhesive solution is given by:

with the length:

$$
\begin{aligned}
d(\varepsilon) & =d^{\mathrm{na}}(\varepsilon)-\Delta l_{c}(\varepsilon), \\
F_{N}(\varepsilon) & =F_{N}^{\mathrm{na}}(\varepsilon)-k_{N}^{\mathrm{na}}(\varepsilon) \Delta l_{c}(\varepsilon),
\end{aligned}
$$

$$
\Delta l^{2}=-\frac{4 \pi b \Delta w}{\mathrm{~d} k_{N}^{\text {na }} / \mathrm{d} b} .
$$

Using the non-adhesive solution obtained above, thus inserting Eq. (25) into Eq. (27) we obtain:

$$
\Delta l_{c}(\varepsilon)=d_{0} \sqrt{\frac{\varepsilon \gamma_{1}^{\prime 2}(\varepsilon)}{\gamma_{1}^{\prime \prime}(\varepsilon) \gamma_{2}^{\prime}(\varepsilon)-\gamma_{2}^{\prime \prime}(\varepsilon) \gamma_{1}^{\prime}(\varepsilon)}} .
$$


The critical state, for which the contact loses its stability, can be derived from the relations:

$$
\frac{\mathrm{d} F_{N}}{\mathrm{~d} \varepsilon}=2 E^{*} h a\left\{\gamma_{2}^{\prime}(\varepsilon)-\frac{d_{0}}{h} \frac{\mathrm{d}}{\mathrm{d} \varepsilon}\left[\gamma_{2}^{\prime}(\varepsilon) \sqrt{\frac{\varepsilon}{\gamma_{1}^{\prime \prime}(\varepsilon) \gamma_{2}^{\prime}(\varepsilon)-\gamma_{2}^{\prime \prime}(\varepsilon) \gamma_{1}^{\prime}(\varepsilon)}}\right]\right\}=0,
$$

for fixed loads, and:

$$
\frac{\mathrm{d} d}{\mathrm{~d} \varepsilon}=h\left\{\gamma_{1}^{\prime}(\varepsilon)-\frac{d_{0}}{h} \frac{\mathrm{d}}{\mathrm{d} \varepsilon}\left[\gamma_{1}^{\prime}(\varepsilon) \sqrt{\frac{\varepsilon}{\gamma_{1}^{\prime \prime}(\varepsilon) \gamma_{2}^{\prime}(\varepsilon)-\gamma_{2}^{\prime \prime}(\varepsilon) \gamma_{1}^{\prime}(\varepsilon)}}\right]\right\}=0,
$$

for fixed grips.

In normalized variables $d / h$ and $F_{N} /\left(2 E^{*} h a\right)$ the solution obviously only depends on dimensionless surface energy $d_{0} / h$ and ratio $\varepsilon$.

In Fig. 9 the normalized relations between indentation depth and normal force for different values of $d_{0} / h$ are shown for the conical and the spherical concave indenter (using the approximations of the non-adhesive solutions obtained in Section 2).

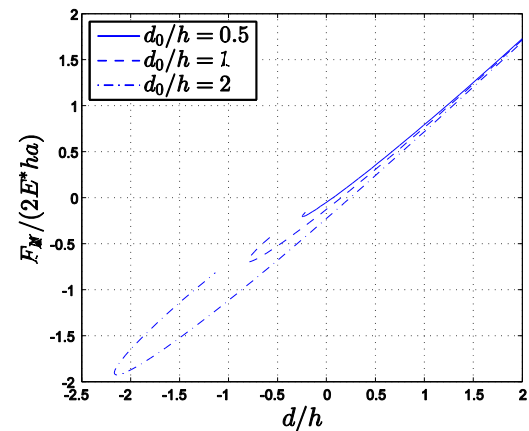

(a)

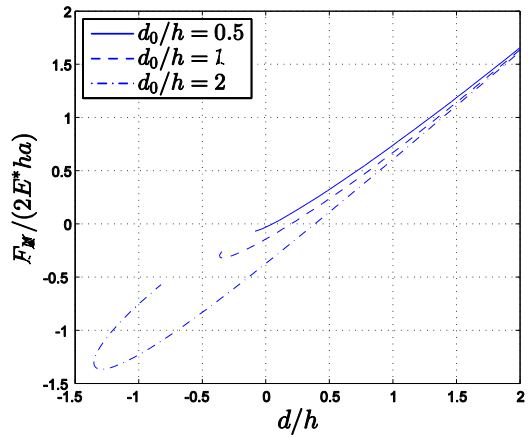

(b)

Fig. 9 Normalized relation between indentation depth $d / h$ and normal force $F_{N} /\left(2 E^{*} h a\right)$ for different values of the surface energy $d_{0} / h=\sqrt{2 \pi a \Delta w / E^{*} h^{2}}$ for the JKR-adhesive normal contact of a concave indenter with the profile shape being: (a) conical and (b) spherical

In Fig. 10 we give the results for the critical state for both indenters. The values of $\varepsilon$ and the normal force - normalized for comparison on $F_{0}=\sqrt{8 \pi a^{3} E^{*} \Delta w}=2 E^{*} a d_{0}$ - for which the contact loses stability and detaches, are given in dependence of the dimensionless surface energy for both fixed grips and fixed loads. Obviously $\varepsilon_{c r i t}$ is always larger for the fixedgrips-regime. Interestingly the critical normal force, often called adhesive or pull-off force, almost does not depend on the regime, as the curves for the fixed grips and fixed loads overlap for both the conical or spherical concave indenter. Also it is visible that for $d_{0} \gg h$ the concave indenters can achieve pull-off forces significantly larger (up to 50\% for conical and $60 \%$ for spherical profiles) than $F_{0}$, i.e. the pull-off force for a flat cylindrical punch with radius $a$. 


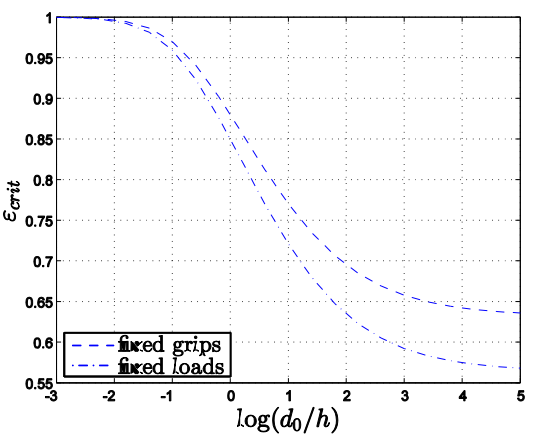

(a)

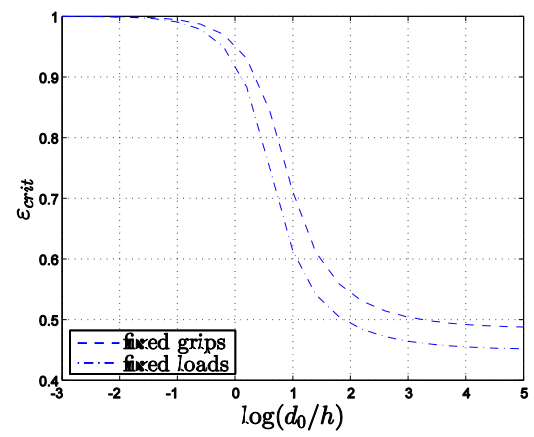

(c)

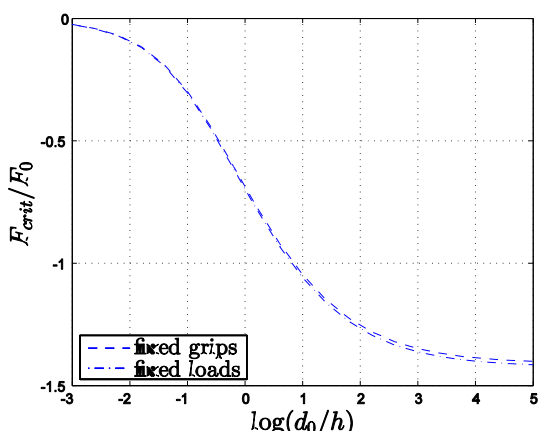

(b)

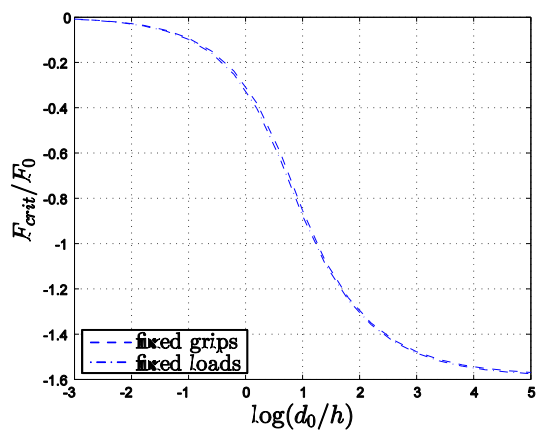

(d)

Fig. 10 Dependence of critical ratio $\varepsilon_{c r i t}$ and normalized adhesion force $F_{c r i t} /\left(2 E^{*} a d_{0}\right)$ on normalized surface energy $d_{0} / h$ with fixed grips and fixed loads for the JKR-adhesive normal contact of a conical or spherical concave punch. (a) $\varepsilon_{\text {crit }}$, conical, (b) $F_{c r i t} /\left(2 E^{*} h a\right)$, con., (c) $\varepsilon_{\text {crit }}$, spherical (d) $F_{c r i t} /\left(2 E^{*} h a\right)$, spher.

\section{CONCLUSIONS}

Based on the formalism in [15], the JKR-adhesive normal contact problem with a ringshaped contact area has been solved for the annular flat and spherical or conical concave indenter. The non-adhesive problem was solved using fast boundary element simulations and the solutions were approximated by simple analytic expressions. After that the adhesive solutions can be obtained analytically from the non-adhesive one without problems. The method used can be applied to different axisymmetric concave indenters as well.

We find that the annular flat punch will always start to detach at the outer boundary. The pull-off forces for both concave punch shapes almost do not depend on the pull-off boundary regime, i.e. fixed grips or fixed loads, and can be significantly larger than the adhesion force on a cylindrical flat punch. We also gave solutions for intermediate values of the ratio of contact radii, in which case the asymptotic results obtained in the literature - with this ratio being either close to zero or unity - do not overlap each other very well. 


\section{REFERENCES}

1. Spolenak, R., Gorb, S., Gao, H., Arzt, E., 2005, Effects of Contact Shape on the Scaling of Biological Attachments, Proceedings of the Royal Society of London, Series A, 461, pp. 305-319.

2. Johnson, K.L., Kendall, K., Roberts, A.D., 1971, Surface Energy and the Contact of Elastic Solids, Proceedings of the Royal Society of London, Series A, 324, pp. 301-313.

3. Gubenko, V.S., Mossakovskij, V.I., 1960, Pressure of an Axially Symmetric Circular Die on an Elastic Half Space, Prikladnaya Matematika i Mekhanika, 24, pp. 334-340.

4. Collins, W.D., 1962, On Some Triple Series Equations and Their Applications, Archive for Rational Mechanics and Analysis, 11, pp. 122-137.

5. Collins, W.D., 1963, On the Solution of Some Axisymmetric Boundary Value Problems by Means of Integral Equations. VIII. Potential Problems for a Circular Annulus, Proceedings of the Edinburgh Mathematical Society, Series 2, 13, pp. 235-246, doi: 10.1017/S0013091500010889

6. Borodachev, N.M., Borodacheva, F.N., 1966, Penetration of an Annular Stamp into an Elastic HalfSpace, Mekhanika Tverdogo Tela, 1(4), pp. 158-161.

7. Shibuya, T., Koizumi, T., Nakahara, I., 1974, An Elastic Contact Problem for a Half Space Indented by a Flat Annular Rigid Stamp, International Journal of Engineering Science, 12, pp. 759-771.

8. Gladwell, G.M.L., Gupta, O.P., 1979, On the Approximate Solution of Elastic Contact Problems for a Circular Annulus, Journal of Elasticity, 9, pp. 335-348.

9. Roitman, A.B., Shishkanova, S.F., 1973, The Solution of the Annular Punch Problem with the Aid of Recursion Relations, Prikladnaya Mekhanika, 9(7), pp. 37-42.

10. Antipov, Y.A., 1989, Analytic Solution of Mixed Problems of Mathematical Physics with a Change of Boundary Conditions over a Ring, Mechanics of Solids, 24(3), pp. 49-56.

11. Barber, J.R., 1976, Indentation of the Semi-Infinite Elastic Solid by a Concave Rigid Punch, Journal of Elasticity, 6, pp. 149-159.

12. Shibuya, T., 1980, Indentation of an Elastic Half-Space by a Concave Rigid Punch, ZAMM Zeitschrift für Angewandte Mathematik und Mechanik, 60, pp. 421-427.

13. Barber, J.R., 1974, Determining the Contact Area in Elastic Indentation Problems, Journal of Strain Analysis, 9, pp. 230-232.

14. Kesari, H., Lew, A.J., 2012, Adhesive Frictionless Contact between an Elastic Isotropic Half-Space and a Rigid Axi-Symmetric Punch, Journal of Elasticity, 106, pp. 203-224.

15. Argatov, I.I., Li, Q., Pohrt, R., Popov, V.L., 2016, Johnson-Kendall-Roberts Adhesive Contact for a Toroidal Indenter, Proceedings of the Royal Society of London, Series A, 20160218, doi: 10.1098/rspa.20160218.

16. Pohrt, R., Li, Q., 2014, Complete Boundary Element Formulation for Normal and Tangential Contact Problems, Physical Mesomechanics, 17(4), pp. 334-340.

17. Kendall, K., 1971, The Adhesion and Surface Energy of Elastic Solids, Journal of Physics D: Applied Physics, 4, pp. 1186-1195. 